CERN LIBRARIES, GENEVA

CM-P00062402

CERN-TH. 4629

March 1987

\title{
NON-CHAOTIC KALUZA-KLEIN COSMOLOGY
}

Lars Gerhard Jensen

CERN, Geneva, Switzerland

\section{A B S T R A C T}

We investigate the evolution of higher dimensional, homogeneous, anisotropic cosmologies. The natural extension to higher dimensions of the 3-dimensional mixmaster cosmologies are analysed, and it is found -- contrary to the 3-dimensional case -- that the higher dimensional cosmologies exhibit no chaotic behaviour. 


\section{INTRODUCTION}

In the past few years higher dimensional cosmologies have received a great deal of attention [1]. This is due to the theoretical possibility of a unification of the fundamental forces in a higher dimensional space-time. In these theories the extra dimensions are usually assumed to compactify at some energy scale of the order of the Planck mass, while the remaining dimensions expand into our observable universe. In the type of models commonly considered, the extra dimensions are curled up in a maximally symmetric n-sphere. This rather arbitrary assumption is usually made because of the simple geometry of maximally symmetric spaces. It is well known from 3+1 dimensions that even if one considers a less symetric geometry, there are interesting models, such as the mixmaster cosmologies [2], which can evolve into our observable universe but which, close to the initial singularity, enter an infinite succession of Kasner epochs during which the three scale factors oscillate with arbitrarily large amplitude and frequency. It is also well known that this type of chaotic behaviour is exhibited in the most general (inhomogeneous) cosmologies close to the initial singularity [3]. Recently it has been shown that the same result is true for spatial dimensions between four and nine, and that the chaotic behaviour disappears for dimension ten and above [4]. For $n+1$ dimensional cosmology with $n \geq 10$, the general approach to the singularity is the generalized Kasner solution. In this case the universe enters a finite succession of Kasner epochs, of which the last one is stable and determines the approach to the singularity. For homogeneous cosmologies the problem of the approach to the singularity is still unsolved for $4 \leq \mathrm{n} \leq 9$, where $\mathrm{n}$ is the spatial dimension. Owing to the constraints imposed by the structure constants it is expected that even for $4 \leq n \leq 9$ the general approach to the singularity is the Kasner solution, i.e. a finite succession of Kasner epochs. In this paper, we shall show that this is indeed the case for a large class of homogeneous cosmologies. All the examples of models previously examined in the literature [5] fall within this class, and our result -- obtained in a simpler way -agrees in each case with those obtained in the literature. 


\section{HOMOGENEOUS COSMOLOGY}

The extension to $n+1$ dimensions of the usual $3+1$ dimensional Bianchi models consists of the cosmologies with a one-parameter family of n-dimensional spatial hypersurfaces with a Lie Group acting simply transitively, i.e. the group motion may carry any point into any other point. The Lie Group $G$ has $n$ generators $\mathrm{x}_{\mathrm{a}^{\prime}}$ $a=1, \ldots, n$ and corresponding invariant forms $e^{a}=e_{\mu}^{a} d x_{\mu}, \mu=1, \ldots, n$, which satisfy $d e^{a}=-1 / 2 c_{b c}^{a} e^{b} \Lambda e^{c}$, where $c_{b c}^{a}$ are the structure constants of $G$. We shall assume that the metric is diagonal in the $e^{a}$-basis:

$$
d s^{2}=d t^{2}-R_{a}^{2}\left(e^{a}\right)^{2}
$$

Then we find the following $0-0$ and a-a Einstein equations in vacuum:

$$
\begin{aligned}
& \sum_{a}\left(\frac{\dot{\mathrm{R}}_{a}}{\mathrm{R}_{\mathrm{a}}}\right)^{\cdot}+\left(\frac{\dot{\mathrm{R}}_{\mathrm{a}}}{\mathrm{R}_{\mathrm{a}}}\right)^{2}=0 \\
& \frac{1}{2 \sqrt{g}}\left(\sqrt{g} \frac{\dot{\mathrm{R}}_{\mathrm{a}}}{\mathrm{R}_{\mathrm{a}}}\right)=-\mathrm{P}_{\mathrm{a}}^{\mathrm{a}} \quad \text { (no sum), }
\end{aligned}
$$

where $g=R_{a}^{2} \ldots R_{n}^{2}$ and $P_{a b}$ is the n-dimensional Ricci tensor. We find that

$$
P_{i}^{i}=\frac{1}{2}\left(\frac{R_{C}^{2}}{R_{b}^{2} R_{d}^{2}}\left(C_{d i}^{C}\right)-\frac{1}{2} \frac{R_{i}^{2}}{R_{C}^{2} R_{d}^{2}}\left(C_{C d}^{i}\right)^{2}+R_{i}^{-2} C_{d i}^{C} C_{C i}^{d}+2 R_{d}^{-2} C_{C d}^{C} C_{i d}^{i}\right) \quad \text { (no sum over } i \text { ) }
$$

Now, if the right-hand side of Eq. (1b) can be ignored, then we recover the famous Kasner solution: taking the trace of Eq. (1b) we find $g \sim t^{2}$. Using this again in Eq. (1b) we find that $R_{a} \sim t^{p_{a}}$ with $\Sigma p_{a}=1$. From Eq. (1a) it then follows that $\Sigma \mathrm{p}_{\mathrm{a}}^{2}=1$; this is the Kasner solution.

A closer look at eqs. (1) shows that the Kasner solution is a good approximation as long as $t^{2} P_{a}^{a}$ remains small for small times $t$. We notice that this is always 
the case for the two last terms in $t^{2} \mathrm{P}_{a}^{a}$ since these have the form $t^{2\left(1-p_{C}\right)}$. The first two terms, however, have the form $t^{2 \alpha_{a b c}}$ with $\alpha_{a b c}=1+p_{a}-p_{b}-p_{c}$ and could possibly be negative. It is therefore interesting to investigate which constraints one must impose to demand that the $\alpha_{a b c}$ be positive. If all the $\alpha_{a b c}$ remain positive for arbitrary small times, then the approach to the singularity is that of the Kasner solution, while if negative $\alpha_{a b c}$ exist in $t^{2} P_{i}^{i}$ the corresponding term grows indefinitely for small $t$ and the universe will 'bounce' and enter a new Kasner epoch described by new $p_{a}$. Now, notice from eq. (2) that each term containing $t^{2 \alpha}$ abc is proportional to $\mathrm{c}_{\mathrm{bc}^{\prime}}^{\mathrm{a}}$ i.e. only terms with $\mathrm{c}_{\mathrm{bc}}^{\mathrm{a}} \neq 0$ appear in $\mathrm{P}_{\mathrm{d}}^{\mathrm{d}}$. We may then ask which groups have structure constants such that for a suitable choice of the $p_{a}$ all the $\alpha_{\mathrm{abc}^{\prime}}$ corresponding to non-vanishing $\mathrm{c}_{\mathrm{bc}^{\prime}}^{\mathrm{a}}$ are positive. We shall show that if one among the generators $\mathrm{x}_{\mathrm{a}}$ of $\mathrm{G}$ can find a closed subset of generators -- a subalgebra -- of dimension $d$ with $2 \leq d \leq n-1$, then it is possible to find a region of finite measure in $p$-space such that all the $\alpha_{a b c}$ in $t^{2} P_{i}^{i}$ are positive for $p_{a}$ in this region. This shows that the approach to the initial singularity in this case cannot be chaotic -- after going through a (perhaps large) number of Kasner epochs the universe will eventually bounce into the region with all the $\alpha_{a b c}$ positive. Once described by a set of $\mathrm{p}_{\mathrm{a}}$ from this region the universe evolves for arbitrary small times according to the Kasner solution corresponding to this particular set of $\mathrm{p}_{\mathrm{a}}$.

\section{THE APPROACH TO THE INITIAL SINGULARITY}

Let us assume it is possible to divide the generators $x_{a}$ into a set $\left\{x_{A}\right\}$, $A=1, \ldots, d$ and $\left\{x_{\sigma}\right\}, \sigma=1, \ldots, D$, where $D=n-d$ and where the $x_{A}$ span $a$ d-dimensional subalgebra of $G$, i.e. $C_{A B}^{\sigma}=0$ for all $A, B, \sigma$. Now, we shall provide a set of $\mathrm{p}_{\mathrm{a}}$ such that all $\alpha_{\mathrm{abc}}$ corresponding to non-vanishing $\mathrm{C}_{\mathrm{bc}}^{\mathrm{a}}$ are positive: let $\mathrm{p}_{\mathrm{a}}$ be the Kasner index corresponding to $\mathrm{x}_{\mathrm{a}^{\prime}}$ and define $\mathrm{p}_{\mathrm{A}}=\mathrm{p}, \mathrm{A}=1, \ldots, \mathrm{d}$ and $p_{\sigma}=q, \sigma=1, \ldots, D$, where

$$
\mathrm{p}=\frac{\frac{1}{\mathrm{dD}}+\frac{1}{\mathrm{~d}} \sqrt{\frac{1}{\mathrm{~d}}+\frac{1}{\mathrm{D}}-\frac{1}{\mathrm{dD}}}}{\frac{1}{\mathrm{~d}}+\frac{1}{D}}, \quad \mathrm{q}=\frac{\frac{1}{\mathrm{dD}}-\frac{1}{\mathrm{D}} \sqrt{\frac{1}{\mathrm{~d}}+\frac{1}{\mathrm{D}}-\frac{1}{\mathrm{dD}}}}{\frac{1}{\mathrm{~d}}+\frac{1}{D}}
$$


Here $p$ and $q$ are defined as the solution to the equations $d p+D q=d p^{2}+D q^{2}=1$ (i.e. $\Sigma p_{a}=\Sigma p_{a}^{2}=1$ ). Let us now check the possible values of $\alpha_{a b c}$ :

$$
\begin{aligned}
& \alpha_{\mathrm{ABC}}=1-\mathrm{p}, \quad \alpha_{\mathrm{ABO}}=\alpha_{\mathrm{A \sigma B}}=1-\mathrm{q}, \quad \alpha_{\mathrm{A \sigma Q}}=1+\mathrm{p}-2 \mathrm{q}, \\
& \alpha_{\sigma \mathrm{AQ}}=\alpha_{\sigma \varrho A}=1-\mathrm{p}, \quad \alpha_{\sigma \varrho \tau}=1-\mathrm{q} .
\end{aligned}
$$

These are all positive since $q<p<1 ; \alpha_{\sigma A B}$ does not appear in $t^{2} P_{i}^{i}$ since $C_{A B}^{\sigma}=0$. Now, since all the $\alpha_{a b c}$ are positive for this particular choice of $p_{i}$ they are also positive for a whole surrounding region I of finite measure in p-space. This shows that the region corresponding to all the $\alpha_{a b c}$ being positive has finite measure. Therefore, we can conclude that models which allow for diagonal solutions, and for which the generators can be split as devised above, are non-chaotic and the general approach to the initial singularity is the Kasner solution. All the models already analysed allow such a split of the generators [5], so it follows from our simple argument that these are non-chaotic, in agreement with the earlier analysis.

Any Lie algebra $L$ can be split into two parts $L=Q+M$, where $M$ and $Q$ are subalgebras with $Q$ semisimple and $M$ solvable with $[M, Q] \subset M[6]$. If neither $Q$ nor $M$ are trivial, then the generators of $L$ may be chosen according to the split into $Q$ and $M$, and it follows from our result, that any existing diagonal solution close to the singularity is the (non-chaotic) Kasner solution. If $Q$ is trivial then $L=M$ is solvable, so $L \neq[L, L]$, i.e. $L$ may be split into $L^{\prime}=[L, L]$ plus remaining generators, and our result shows also in this case that the general solution is nonchaotic. Finally if $\mathrm{L}=Q$, then $\mathrm{L}=\mathrm{L}, \oplus \ldots \oplus \mathrm{L}_{\mathrm{k}}$ with $\mathrm{L}_{i}$ being simple. Obviously if $k \geq 2$ we may split the generators and draw the same conclusion. If $k=1$, then $L$ is simple; but the only simple, real algebras without subalgebras are SO(3) and $\mathrm{SO}(2,1)$, i.e. the Bianchi models IX and VIII. These are therefore the only candidates for chaotic behaviour (it is well known that these models exhibit the chaotic mixmater behaviour close to the singularity). The natural candidates as higher dimensional mixmaster cosmologies, namely the higher dimensional simple Lie groups, all contain subalgebras, i.e. it is possible to split the generators as divised 
above. Therefore even for these groups the chaotic behaviour -- which is characteristic for the 3-dimensional simple groups -- is absent.

In conclusion, we have shown that no obvious generalization to higher dimensions of the usual 3-dimensional diagonal mixmaster cosmologies exist. We have shown that whenever diagonal solutions exist they will eventually evolve into the Kasner solution corresponding to a set of $\mathrm{p}_{\mathrm{a}}$ with all the $\alpha_{\mathrm{abc}}$ positive.

Finally, thanks are extended to the members of the Theory Division at CERN for their hospitality and to Jaime Stein-Schabes. 


\section{REFERENCES}

[1] S. Randjbar-Daemi, A. Salam and J. Strathdel, Phys. Lett. 135B (1984) 388;

D. Sahdev, Phys. Lett. 137B (1984) 155;

E.W. Kolb, D. Lindley and D. Seckel, Phys. Rev. D30 (1984) 1205;

E.W. Kolb, Cosmology and extra dimensions, FERMILAB Pub 86-138 (1986).

[2] V.A. Belinskii, I.M. Khalatnikov and E.M. Lifshitz, Adv. Phys. 19 (1970) 525;

C.w. Misner, Phys. Rev. Lett. 22 (1969) 1071;

J.D. Barrow and F.J. Tipler, Phys. Rep. $\underline{56}$ (1979) 372.

[3] V.A. Belinskii, I.M. Khalatnikov and E.M. Liphitz, Adv. Phys. 31 (1982) 631.

[4] J. Demaret, M. Henneaux and P. Spindel, Phys. Lett. B164 (1985) 27;

A. Hosoya, L.G. Jensen and J.A. Stein-Schabes, Nucl. Phys. B283 (1987) 657.

[5] T. Furusawa and A. Hosoya, Prog. Theor. Phys. 73 (1.985) 467;

J.D. Barrow and J.A. Stein-Schabes, Phys. Rev. D32 (1985) 1595;

A. Tomiatsu and H. Ishihara, Gen. Relativ. Gravitation 18 (1986) 161;

H. Ishihara, Prog. Theor. Phys. 74 (1985) 490;

P. Halpern, Phys. Rev. D33 (1986) 354.

[6] V.S. Varadarajan, Lie groups, Lie algebras and their representations (PrenticeHall, Englewood Cliffs, NJ, 1974). 